\title{
Ein Beitrag zur Kenntnis der zentralen Vestibularisbahn.
}

$$
\text { Von }
$$

\section{Isao Yoshida.}

(Aus dem Anatom. Institut d. mediz. Fakult. zu Okayama).

Mit 3 Figuren anf Tafel $\mathrm{XV}$.

Der Deit erssche Kern steht topographisch-auatomisch wie ein Torwächter auf der Grenze zwischen dem Kleinhirn einerseits und der Oblongata und der Brücke andererseits.

Er empfāngt seine zentripetalen Erregungen auf dem Wege des Nervus vestibularis, sei es nun, dass Axone der Zellen des Ganglion Scapr ae selbst oder nur ihre Kollateralen an seine Zellen herantreten. Wie bekannt, sendet er abwärts seine Faøern bis ins Rückenmark hinein, aufwärts in den Hirnstamm, um die Augeumuskelkerne zu beeinflussen.

Von vielen Autoren ist die Existenz der zerebellopetalen Verbindung des Deit e $r$ schen Kerns mit dem Kleinhirn angegeben. Vor Kurzem gelang es mir, mit der $\mathrm{Nisslschen} \mathrm{tigrolytischen} \mathrm{Methode} \mathrm{diese} \mathrm{Verbindung} \mathrm{zu} \mathrm{bestätigen.}$ Und zwar sah ich nach Zerstōrung der verschiedenen Abschnitte des Kleinhirns im Deitersschen Kern zahlreiche stark degenerierte Zellen. Darüber habe ich") schon eingehend nitgeteilt.

Kohnst a m m${ }^{\text {) }}$ fand nach halbseitiger Durchschneidung des Zervikalmarks die Zellen des genannten Kerns fast restlos auf der operierten Seite tigrolytisch verāndert.

Wenn; wie Kohnstamm sagt, die Zellen des Deitersschen Kerns nach Durchschneidung des Rūckenmarks sicherlich fast sämtlich degenerieren, so müssen wir annehmen, dass sich das Axon dieses Kerns dichotomisch teilt, und dass der eiue Ast ins Rūckenmark und der andere ins Kleinhirn geht, oder dass die Zellen dies Kerns ihre Axone ins Rückenmark, und ihre Kollateralen ins Kleinhirn gehen lassen, oder umgekehrt.

1) Yosh ida, Okayama-Igakkwai-Zasshi, 1924.

2) Kohnst amm, Arch. f. Psych. u. Nerv. 1899, p. 681. Monatschr. f. Psych. u. Neur. 1900. 
Vorliegender Versuch wurde vorgenommen, um diese Frage zu lösen.

Kohnstamm: untersugchte nach Hemisektion zwischen 1. und 2: Zervikalsegment beim Kaninchen mit der Nisslschen Methode die Vestibulariskerne. Er sagt, dass der grosszellige dorsofrontale Abschnitt des Deitersschen Kerns auf der operierten Seite , fisst ausschliesslich " degenerierte Zellen führt, wïhrend auf der anderen Seite fast nur der ventrale Teil dieses Abschnittes viel weniger zahlreiche degenerierte Zellen zeigt, und dass „, der ventrokaudale Abschnitt des Deitersschen Kerns " auf beiden Seiten viele degenerierte Zellen enthält. Der letztere Abschnitt entapricht zum Teil dem zerebralen Teil der Radix sp n. acustici, zum Teil vielleicht auch dem äusseren Gebiet des Nucl. triangulario, welches gröasere Zellen führt.

Was das Verhalten des übrigen Abschnittes des Nucl. triang. d.h. des eigentlichen Nucl. triang. anbetrifft, so gibt er darüber nichts an.

Bezüglich des Bechterewschen Kerns sagt er, daos dieser Kern ganz frei von Degeneration bleibt.

Betreffs der Nissl-Untersuchung des Deitersschen Kerns nach der Rückenmarkshemisektion finden wir in der Literatur ausserdem noch die Arbeit von Lloyd2). Dieser Autor bestätigte im Deitersschen Kern der operierten Seite eine Chromatolyse. Über die Ausdehnung der Degeneration kann ich leider nichts wissen, da das Original mir nicht zugänglich ist.

\section{Eigene Untersuchung.}

Ich operierte 5 Kaninchen, um die oben erwähnte Angabe von Kolınsta m m nachzuprüfen. Nur ein einziges von ihnen überlebte die Operation.

Das Operationsverfahren ist kurz folgendes.

Nach der Freilegung der Membrana obturatoria wurde die letztere längs ihres unteren Randes geschnitten. Der hintere Teil des Atlas wurde mit einer. Zange entfernt. Nun trat das Rückenmark offen zu Tage. Es wurde an der linken Hälfte mit einem kleinen Messer quer durchschnitten. Unmittelbar nach der Operation wurde die Atmung des Tieres plötzlich sehr schlerht. Es lag elendiglich auf der operierten Seite. Ungefähr nach 8 Tagen verbesserten sich . diese Symptome nach und nach.

1) Kohnst a mm, loc. cit.

2) Lloyd, Citat nach Neur. Zentralbl. 1900, p. 452. 
11 Tage nach der Operation wurde das wiederhergestellte Tier durch die intravitale Durchspülungsfixation getötét.

Die Autopsie ergab auf der linken Seite eine kleine Iäsion oberhaḷb des 2,. Zervikalnerven, d. h. im unteren Teil des 1 . Zervikalsegmentes. Die rechte Hälfte des Halsmarks ist ganz unversehrt; ebenso die proximalen Teile.

Gehirn und Halsmark wurden im Alkohol gehärtet, im Celloidin eingebettet, in Frontalserienschnitte von $25 \mu$ Dicke zerlegt, und diese mit Thionin gefärbit:

Beim Durchsehen der Serienschnitte erwiesen sich die folgenden Bezirke des Halsmarks auf ungefähr 25 Schnitten als zerstörte Stellen.

1. Der ganze linke Seitenstrang.

2. Der linke Vorderstrang mit Ausnahme der medialen Randzone (d. h. des Vorderstranggrundbündels).

3. Der laterale Teil des linken Burdachschen Stranges.

4. Der grösste laterale Teil der grauen Substanz der linken Seite.

Mit kurzen Worten ist die Tinke Hälfte des Halsmarks mit Ausnahme des kleinen medialen Abschnittes auf der Höhe des unteren Teils des 1. Zervikalsegmentes zer'stört (Fig. 1). In der anderen Hälfte des Halsmarks sowie im Gehirn findet man keine Läsion.

Als die Folge dieser Läsion fand ich ausser den Vestibulariskernen noch in der Formatio reticularis des Halsmarks, der Oblongata und der Brücke, im Nucleus reticularis raphes sowie im roten Kern degenerierte Zellen. Im folgenden werde ich doch nur die Veränderung der Vestibulariskerne beschreiben.

Nucleus triangularis bleibt anf beiden Seiten ganz intakt. Nucleus intercalatus Staderini ebenso. Aber der laterale Teil des Nucl. triang. d. h. der Triangularisanteil des Deitersschen Kerns (Fuse) enthält auf beiden Seiten grosse und mittelgrasse degenerierte Zellen.

Die graue Substanz der Radix sp. n. acustici zeigt in ihrem zerebralsten Abschnitt auf beiden Seiten grosse und mittelgrosse degenerierte Zellen. Dagegen ist der grössere spinale Teil frei von Degeneration.

Betreffs des Deitersschen und Bechterewschen Kerns fand ich genau dasselbe wie Kohastanm.

Der Deiterssche Kern der operierten Seite enthält-nämlich fast ausschliesslich degenerierte Zelken. Die Degeneration ist ganz hochgradig (Aufblähung, blasses Aussehen u. Kernverlagerung) und betrifft grosse wie mittelgrosse Zellen 
(Fig. 2 u. 3). Man kann nur einige Zellen als ganz gesund betrachten, und zwar vorwiegend im ventralen Abschnitt des Kerns. Auf der anderen Seite sind die Zellen des kleineren ventralen Teils dieses Kerns nur vereinzelt doch stark tigrolytisch verändert, aber der übrige grössere Teil zeigt keine einzige degenerierte Zelle:

Im Bechterewschen Kerne sind auf beiden Seiten keine degenerierten Zellen zu finden.

Aus dem oben erwähnten Befunde geht wohl hervor, dass die Zellen des Deitersschen Kerns mit Ausnahme des ventralen Abschnittes fast sämtlich mit dem Rückenmark in Verbindung stehen.

Andererseits ist es auch ganz sicher, dass der Deiter ssche Kern zahlreiche Axone bzw. Kollateralen ins Kleinhirn entsendet, da ich nach Kleinhirnläsion zahlreiche hochgradig degenerierte Zellen in diesem Kern, besonders in seinem dorsalen Abschnitt, konstatieren konnte.

Deshalb müssen die zahlreichen Zellen dieses Kerns sowohl ins Rückenmark als auch ins Kleinhirn ihre Fasern entsenden, um mit diesen beiden Teilen in Verbindung zu stehen. Damit diese zweifache Verbindung dieser Zellen möglich ist, kann man annehmen, dass die Axone der Zellen sich dichotomisch teilen und ihre Äste einerseits nach dem Rückenmark andererseits ins Kleinhirn senden. Allerdings ist es möglich, dass diese Doppelverbindung nicht durch die dichotomische Teilung der Axone, sondern durch die Abgabe der Kollateralen stattfindet.

Aber Ramon y Cajal1), dem wir zahlreiche Arbeiten über die Struktur der Nervenzellen auf Grund der Golgischen Untersuchung verdanken; hat gezeigt, dass sich die Axone des Deitersschen Kerns, wenn auch erst im Haubenfeld der Oblongata, dichotomisch teilen. Daher kann man mit Recht annehmen, dass sich die Axone aus dem Deitersschen Kern, wenigstens zum Teil, dichotomisch teilen und einerseits nach dem Rückenmark anderseits ins Kleinhirn ziehen.

Diese Annalıme wird um so mehr begründet sein, als die Zellen des Deitersschen Kerns hinsichtlich des Degenerationsgrades ganz gleicherweise auf die Rückenmarkshemisektion wie auf die Kleinhirnläsion reagieren.

Jedenfalls scheint es mir sehr interessant, dass ein und dasselbe Neuron sowohl ins Rückenmark wie ins Kleinhirn hin Erregungen leitet.

1) Ramon y Ca ia l, Beitrag z. Studium d. Medulıa oblon. 1896, p. 69 Erklärung zur Fig. 19. 
Selbotverständlich ist es auch möglich, dass der eine Ast von den dichotomisch geteilten Axon oder seine Kollateralen nach den Augenmuskelkernen ziehen, wenn die Verbindung des Deitersschen Kerns mit den letzteren sicher ist.

Der ventrale Abschnitt des Deitersschen Kerns und der zerebralste Abschnitt der grauen Substanz der Radix spinalis n. acustici zeigen, im Einklang nit Kohnstam m, auf beiden Seiten vereinzelte degenerierte Zellen. Somit ist es sicher, dass clie Zellen dieser Abschnitte ihre Axone baw. Kollateralen ins Rückenmark entsenden, und zwrar doppelseitig.

Über den Wert des negativen Befundes bei der $\mathrm{N}$ isslschen tigrolytischen Methode verweise ich auf meine frühere Arbeit:

Der Nucleus triang. und der Bechterewsche Kern zeigen keine degenerierten 7ellen, trotzle:n die Untersuchung nach ol timaler postoperativer Lebensdanier ausgeführt worden ist. In Bezug auf den Bechterewschen Kern erzielte auch Kohnstamm ganz gleiches Resultat. Auf Grund dieses Befundes kann man sagen, dass diese Kerne ins Rückenmark keine Axone bzw. grobkalibrigen Kollateralen senden.

Es ist interessant, dass der Deiterssche Kern, welcher spärliche wenn auch grōsere Zellen enthält, nicht nur mit dem Kleinhirn und den Augennuskelkernen, sondern auch mit dem Rückenmark in Verbindung steht, während der Bechterewsche Kern, welcher aus kleineren Zellen besteht, sich nur mit den ersteren beiden Hirnteilen verbindet: Begreiflicherweise bedarf es grosser Ausbildung der einzelnen Elemente, um mit wenigen Elementen eine grössere Rolle zu spielen.

Die genannte Tatsache steht im Einklang mit der Annahme, dass im allgemeinen lāngere Axone gröaseren Zellen und kürzere kleinern entspringen.

Ferner bestätigt mein Befund die Ansicht, dass nur der eine Ast des dichotomisch geteilten Axon durchtrenut zu werden braucht, damil die $\mathrm{Ni}$ iss degeneration der Ursprungszelle zu Stande kommt.

\section{Schluss.}

1. Der Deiterssche Kern steht in Verbindung mit dem Rückenmark und mit dem Kleinhirn.

2. Die Axone dieses Kerns teilen sich wanigatens zum Teil dichotomisch, und

1) Yoshida, loc. cit. 
der eine Ast von dem dichotomisch geteilten Axon begibt sich ins Rückenmark und der andere Ast ins Kleinhirn.

3. Deshalb: leitet ein und dasselbe Neuron Erregungen sowohl ins Rückenmark wie ins Kleinhirn hin.

4. Der ventrale Abschnitt des Deitersschen Kerns und der zerebralste Absehnitt der grauen Substanz der Radix spinalis nervi acustici stehen in Verbindung mit dem Rückenmark, und zwar ungekreuzt oder gekreuzt.

5. Der Bechterewsche Kern entsendet keine Axone ins Rückenmark.

6. Der Nucleus triangularis entsendet keine Axone ins Rückenmark.

Es erübrigt sich nur noch, Herrn Prof. Kôsaka für die mir bei dieser Arbeit erwiesene Unterstützung vèrbindlichst zu danken.

\section{Verzeichnis der Abbildungen (Tafel XV).}

Fig. 1 Mikrophotographie der Verletzungsstelle des Halsmarks.

Fig. 2 Mikrophotographie der Deitersschen Kerne. Der Kern ist auf der linken Seite degeneriert.

Fig. 3 Mikrophotographie der degenerierten Zellen des Deitersschen Kerns.

$$
\begin{aligned}
& \text { Crst }=\text { Corpus restiforme } \\
& \text { vIV }=\text { vierter Ventrikel } \\
& \text { Ndt }=\text { Nucleus dentatus } \\
& \text { ND }=\text { D eiter sscher Kern } \\
& \text { NVI }=\text { Abducenskern } \\
& \text { NVII = Facialiskern } \\
& \text { Nvt }=\text { Nucleus triangularis } \\
& \text { Coa }=\text { Vorderhorn } \\
& \text { Fsla }=\text { Fissura longitudinalis ventralis }
\end{aligned}
$$


FOLIA ANATOMICA JAPONICA, BD. II.

Tu. ac,

TAF. XV.

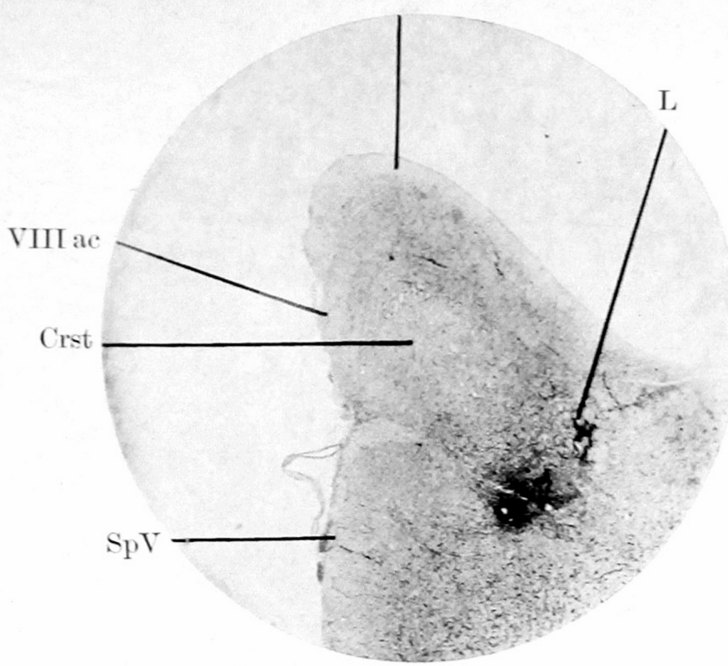

Fig. 1.

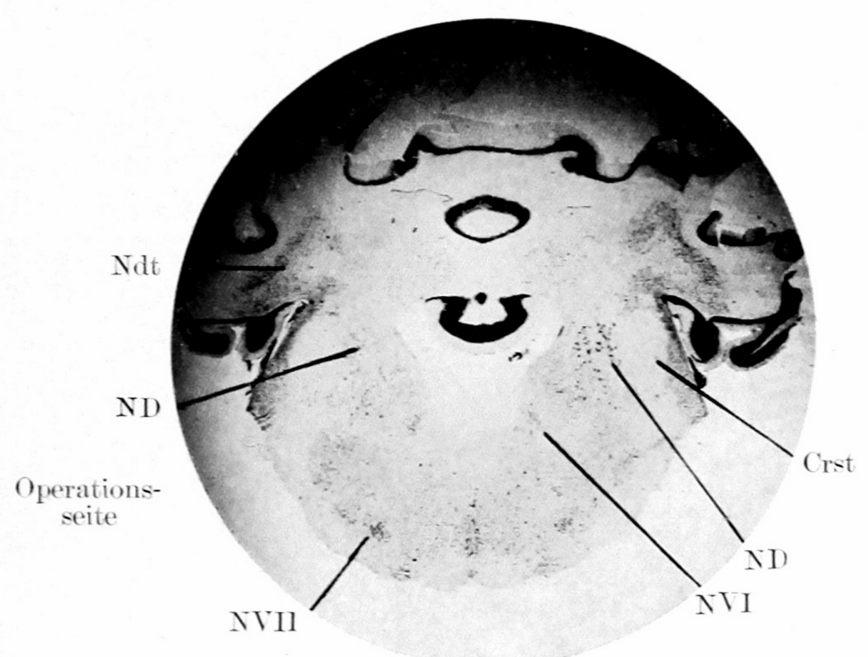

Fig. 2.

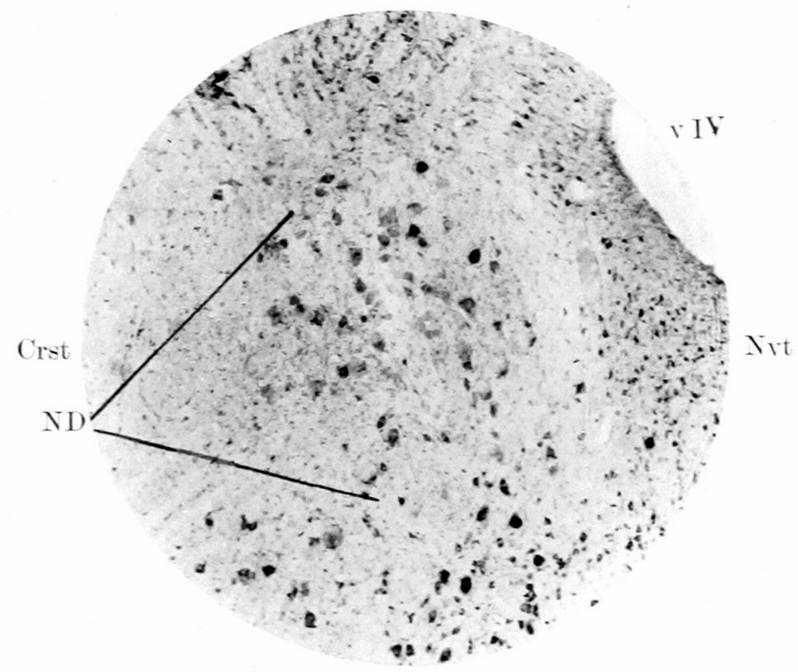

I. Yoshida.

Fig. 3. 